\title{
SCENARI E TENDENZE DELLA PROFESSIONE ACCADEMICA: LA RIVISTA E L'ASSOCIAZIONE A CONFRONTO
}

\author{
Daniele Dalli, Alberto Mattiacci
}

In questo editoriale cerchiamo di far emergere alcuni aspetti della professione accademica e del relativo contesto che il protagonista dell'intervista ha ben presenti per il ruolo e le funzioni che svolge. La Rivista ritiene utile un confronto in tal senso allo scopo di sostenere i soci e i lettori in una fase in cui la normativa e le istituzioni di governo, e per la verità anche molti accademici spontaneamente, stanno contribuendo a trasformare il profilo tradizionale del docente universitario. In tale condizione non sono disponibili modelli di riferimento univoci e affidabili e per questa ragione il punto di vista di Alberto Mattiacci su tali tematiche è particolarmente prezioso.

Dalli. A oltre un anno dalla nomina a Segretario Generale della SIMktg, qual è la situazione della nostra società accademica?

Mattiacci. Direi molto soddisfacente: aumenta la base associativa, sia nei soci accademici che nei partner aziendali, il portafoglio di attività si è arricchito di nuove iniziative (la Scuola per la ricerca, l'Osservatorio Insegnamento del Marketing, la Vetrina Pubblicazioni, per citarne solo alcune), i Master patrocinati sono sempre più numerosi e coprono l'intero territorio nazionale, il nuovo sito inizia a fare numeri interessanti.

Per quanto attiene, poi, alle attività primarie della SIMktg, detto che può sempre farsi meglio, mi pare che vi siano state novità positive di rilievo: il Convegno ha festeggiato il proprio decennale, il Premio si è arricchito dell'importante partnership istituzionale di Centromarca, la Rivista ha cambiato direzione senza traumi e sta procedendo verso importanti nuovi traguardi.

Se devo individuare un punto ancora non soddisfacente è quello dei rapporti con le imprese. Qui siamo ancora lontani dal punto di svolta, nonostante i molti sforzi che la Presidenza sta profondendo e la disponibilità

Mercati e Competitività n. 4, 2013 
delle imprese partner. Di certo l'accentuarsi dell'autoreferenzialità dell'attività accademica in corso di questi tempi non aiuta a migliorare questa situazione, che vede già la difficoltà di confrontarci con un mondo, le imprese, che di per sé non ha come priorità essenziale quella del dialogo con l'Università.

Vorrei esser molto chiaro su questo punto: se passa l'idea che i concorsi si vincono solo (non anche, solo!) con le pubblicazioni scientifiche internazionali, se passa un modello di giovane ricercatore che sta giornate di fronte alle banche dati e ai software econometrici, se vince l'idea che insegnamento e ricerca applicata conto terzi siano solo seccature e distrazioni rispetto all'obiettivo di pubblicare paper, perché mai le imprese dovrebbero essere interessate a noi?

Dalli. Capisco. Peraltro, ho sempre pensato che la battaglia vera è quella della produttività nell'ambito della ricerca e nella selezione del personale. Per troppo tempo la nostra accademia ha fatto poca ricerca e di modesta qualità. Con effetti deleteri sulla didattica e sulla capacità di interessare il mondo delle imprese e delle istituzioni, oltre che l'accademia internazionale. Adesso che si comincia a cambiare si manifesta qualche squilibrio, ma sarebbe bene che i colleghi si facessero una ragione una volta per tutte che la nuova università si bassa su un intenso e qualificato impegno nella ricerca. Da questo punto di vista alla SIMktg queste cose le diciamo da tempo, no?

Mattiacci. Sottoscrivo totalmente quanto affermi e credo che la SIMktg possa e debba giocare un ruolo guida nella evoluzione culturale cui ti riferisci. Confido che in questa transizione la nostra associazione possa fungere da cassa di compensazione fra atteggiamenti opposti che ritengo entrambi errati - il talebanismo e il conservatorismo - riportando un po' di serenità e senso della misura nel nostro ambiente. Ho molte ragioni per essere ottimista in questo senso.

Tornando alla SIMktg mi sento di poter dire che siamo una piccola realtà associativa che, con pochi mezzi, grazie al contributo volontario di moltissimi colleghi, cresce in qualità e quantità delle attività. Credo che anche l'aver ricevuto il Patrocinio del Convegno da parte della Presidenza della Repubblica sia un piccolo, ma significativo riconoscimento della qualità del lavoro compiuto in tutti questi anni.

Dalli. A tuo parere quale ruolo deve svolgere la rivista Mercati e Competitività nell'ambito della SIMktg e più in generale nel panorama delle riviste di area aziendale?

Mattiacci. La rivista ha un ruolo essenziale: senza di essa la nostra associazione non avrebbe senso culturale. Dobbiamo tutti impegnarci, però, a che essa cresca sempre di più in rilevanza e visibilità, sia a livello internazionale, accreditandosi magari come la voce del marketing Made in Italy, 
che a livello nazionale, attraendo l'interesse degli studiosi, giovani in particolare.

Da tempo, come sai, propugno personalmente (con un po' di romantico rammarico) l'abbandono della lingua italiana e la decisa virata internazionale della testata e la sua digitalizzazione. Mi sembra evidente, infatti, che la Rivista sia decisamente posizionata nello spazio della competizione accademica, abbandonata ogni ambizione di rivolgersi a un lettore professionale. Se così è dobbiamo puntare al massimo e nel più breve tempo possibile. Sono convintissimo che la comunità italiana degli studiosi di marketing abbia molto - e di interesse - da dire nel e al mondo. Sono convintissimo che possiamo ambire a immaginare diversi modelli di marketing, non appiattiti su quelli angloamericani e mi piace immaginare che la nostra Rivista ne possa diventare uno strumento di punta.

Dalli. In effetti questo è lo scenario per il futuro e nel presente stiamo lavorando per andare in questa direzione. Anche altre accademie di marketing in Europa e nei paesi emergenti seguono questa strada e la "globalizzazione" del marketing e il pensiero unico Made in the U.S. non è più il paradigma dominante, anzi. In prospettiva, lo dico a beneficio dei ricercatori più giovani, è da tenere presente che il grosso del flusso di citazioni si sposterà verso i paesi che hanno una base economica e accademica in crescita e che "non" hanno il bisogno, né la volontà come appare evidente, di farsi colonizzare culturalmente o professionalmente. È evidente, peraltro, che in tale contesto la lingua del confronto sarà ancora per molto tempo l'inglese. E di certo, ahimé, non l'italiano.

Mattiacci. Concordo e perdonami se torno un attimo sul tema linguistico. È noto che l'inglese, o il globish, sia la lingua predominante, se non esclusiva, della comunicazione scientifica. Data questa evidenza acquisita e dato il posizionamento accademico di Mercati e Competitività, credo non si debba indugiare oltre nel cambiamento di lingua e di distribuzione: l'una senza l'altra funziona poco. Di contro, l'italiano resta la lingua della comunicazione divulgativa (alle imprese e organizzazioni) e didattica: non possiamo, secondo me, abbandonarlo del tutto e perciò occorre che i criteri di abilitazione del futuro tengano in considerazione anche questo fatto.

Dalli. Quali sono le richieste che provengono dalla base associativa e come risponde la Simktg?

Mattiacci. In realtà, a essere sinceri, il funzionamento dell'associazione è di modello push più che pull: a me sembra infatti che, in particolare nel corso di questi ultimi anni, l'attenzione di tutti sia stata comprensibilmente catalizzata dalle molte novità che hanno riguardato il mondo accademico, e che questo fatto abbia incrementato la distrazione e la passività verso l'associazione e altre questioni istituzionali. Mai come nel caso dell'associazionismo culturale, la regola della continuità dell' azione, che tutti noi inse- 
gniamo fra i fondamenti del marketing, è indispensabile: è un mercato, per dirla con espressioni a noi consuete, non spontaneo ma sollecitato.

Per questa ragione cerchiamo sempre di proporre cose nuove e, interpretando gli stimoli dello scenario, che possano essere in armonia con $\mathrm{i}$ cambiamenti della nostra professione. La vetrina pubblicazioni dei soci, per fare un esempio, è stata lanciata anche per favorire la conoscenza della pubblicistica dei colleghi e incrementare le citazioni dei lavori di tutti. La Scuola Sim, per farne un altro, serve a incrementare le competenze di ricerca e comunicazione dei risultati della ricerca medesima. Le Sezioni Tematiche sono state rinnovate per essere maggiormente in linea con i temi del dibattito internazionale. Sono tre esempi di come stiamo cercando di guidare l'associazione: essere strumento per lo sviluppo e il miglioramento individuale del ricercatore in marketing.

Dalli. È vero, in effetti è difficile chiedere oggi a tutti i colleghi, soprattutto quelli che devono fare carriera un impegno assiduo. Quello che però possiamo e dobbiamo chiedere è lo sviluppo di un senso di appartenenza e di solidarietà. Basta poco perché il marketing è uno dei gruppi più dinamici all'interno del nostro settore e anche rispetto ad altri settori nelle materie aziendali. Se riusciamo a condividere un po' di fonti, di risorse e sostenerci a vicenda, ad esempio citando i lavori dei colleghi e gli articoli della Rivista. In poco tempo possiamo riuscire a sviluppare un posizionamento e un ranking di tutto rispetto.

Dalli. La nostra professione si sta modificando: quali sono i profili o gli aspetti di maggiore criticità? E cosa fanno la Simktg e la Rivista per sostenere $i$ colleghi di marketing?

Mattiacci. Io credo che vi siano due criticità principali, entrambe rilevantissime.

La prima è l'autoreferenzialità di cui ho detto prima. Capisco che il modello autoreferenziale sia più rassicurante e lineare di uno che chiama anche al confronto con l'economia reale, ma credo che la chiusura nel circolo accademico sia la risposta più sbagliata alla crisi dell'istituzione universitaria che il Paese (non da solo) sta sperimentando.

In questo concorso di abilitazione, per dire, ci siamo sforzati di mantenere un atteggiamento valutativo flessibile, premiando sempre il merito, sia che esso si concretizzi in un modello lineare - chiamo così quello di chi interagisce con gli operatori, le istituzioni e la realtà economica e professionale al di fuori dell'Università - che circolare - chiamo così chi concentra la propria attività sulla sola ricerca, e in particolare sui paper. È stato nostro dovere procedere in tal modo, perché un Commissario non può valutare il merito solo sulla base di cosa personalmente ritenga giusto e cosa sbagliato, ma deve riuscire a leggere il merito anche interpretando la varietà degli approcci. Tuttavia non posso fare a meno di segnalare le tracce di una decisa virata verso un modello di accademia in cui i ricercatori 
parlano fra loro. Personalmente credo che un professore universitario di marketing dovrebbe essere in grado anche di sedere in un Consiglio di Amministrazione e dire cose che non inneschino reazioni di sgomento e incredulità negli altri consiglieri. Mi chiedo se l'autoreferenzialità che stiamo perseguendo ci porti a questo.

Dalli. Comprendo il tuo punto di vista, soprattutto alla luce della ampiezza di visuale che hai acquisito con la partecipazione all'ASN in qualità di commissario e presidente di commissione. È però da sottolineare che nel modello circolare il dialogo si è comunque ampliato dal contesto nazionale a quello internazionale e che attraverso questo canale i nostri ricercatori si stanno mettendo nelle condizioni di partecipare a un dibattito più ampio rispetto a quello tradizionale che era sostanzialmente limitato al contesto italiano. Se riuscissimo a integrare $i$ due modelli (lineare e circolare) probabilmente la nostra accademia comincerebbe a somigliare a quella di tanti altri paesi e risulterebbe al tempo stesso meno provinciale e più aperta all'esterno.

Mattiacci. La contaminazione dei modelli cui fai riferimento è, secondo me, proprio il punto di arrivo: dobbiamo divenire una comunità di ricercatori che, in quanto tali, sono parte del mondo, che con il mondo dialogano e nel mondo portano idee originali; dobbiamo esser così flessibili da essere capaci, al contempo, di armonizzare quella ricerca con una capacità di essere protagonisti attivi nel Paese: nell'economia reale e nelle aule.

Una seconda criticità è quella di riuscire rapidamente a definire delle regole del gioco, chiare e inequivocabili, pro tempore costanti. A chi fa marketing viene facile indossare i panni del cliente per capire come creare e realizzare valore, no? Allora, se io faccio quest'esercizio e considero come cliente un giovane neolaureato che intenda cimentarsi nella carriera accademica, capisco che lui ha bisogno di: avere un riferimento stimolante (il docente/i mentore e un Dipartimento vivace e proteso alla qualità), sapere su che basi verrà valutato domani, avere un trampolino di lancio verso l'inserimento nella rete internazionale della ricerca e della didattica, potersi sostenere materialmente con il proprio lavoro (l'Università sta tornando ad essere un mestiere per ricchi), eccetera.

Io credo che oggi si vivano delle condizioni che, fatte le debite proporzioni, non si discostino di molto da quelle dell'immediato dopoguerra: siamo di fronte all'esigenza di una ricostruzione e reingegnerizzazione del Paese e quindi anche della sua Università. Il modo giusto, a mio parere, di prendere delle decisioni efficaci al riguardo è declinarle al tempo futuro, e questo significa farsi carico del punto di vista di chi, oggi, è giovane e all'inizio della propria avventura professionale. Diversamente si fa retroguardia e allora la liceizzazione dell'Università è giustificata e la perdita di reputazione sociale della nostra professione pure. 
Mai come oggi noi che possiamo perlomeno tentare di influenzare il domani, dobbiamo porci a servizio di chi verrà dopo di noi e non delle nostre piccole parrocchie attuali.

Dalli. Le componenti del nostro lavoro sono tre: didattica, ricerca e impegno istituzionale (nell'Università e nella società): dal tuo punto di osservazione vedi opportunità, conflitti, tendenze evolutive?

Mattiacci. Come sai ho sempre e in più sedi sostenuto quest'idea di una triplice natura della professione, avversando quella, legittima ma secondo me assurda e autolesionista, che noi si debba fare solo ricerca e che solo quello conti nella valutazione di un accademico. La nostra professione è un cocktail, dove vi sono ingredienti da miscelare in diversa misura. L'ingrediente ricerca è preponderante, forma la base del cocktail e se non è di qualità adeguata guasta il sapore del tutto. Tuttavia non è l'unico.

A me piace porla così: perché mai lo Stato dovrebbe continuare a pagarci uno stipendio? La risposta "per fare ricerca e innovazione" la possono dare solo quelli che scoprono molecole salvavita, materiali straordinari e cose simili; non noi. La nostra risposta di studiosi di marketing dovrebbe secondo me suonare pressappoco così: "per migliorare le persone e la capacità competitiva del nostro Paese, fornendo loro cultura e intelligenza adeguate al mondo di domani e costantemente potenziate da una ricerca continua di qualità". L'utilità sociale dei nostri temi di ricerca, delle nostre "scoperte" e "analisi" non giustifica, secondo me, che uno stipendiato dallo Stato faccia ricerca a tempo pieno, tralasciando insegnamento e impegno istituzionale. Noi serviamo al Paese perché mettiamo costantemente in discussione i nostri saperi e paradigmi, li validiamo e innoviamo con la ricerca e trasferiamo il risultato di tutto ciò ai discenti in maniera efficace e continuamente rinnovata.

Mi ripeto, ma giova: temo che l'autoreferenzialità accademica sarà la sconfitta storica dell'Università italiana, ciò che ci toglierà definitivamente reputazione sociale e percezione d'utilità. Chi la sta perseguendo porterà, se riuscirà nel proprio intento, la responsabilità storica della marginalizzazione sociale dell'istituzione, condividendola con chi, distratto, non ha fatto abbastanza per contrastarla. Credo che su questo fatto tutti dovremo rifletterci su più di quanto non stiamo facendo.

Dalli. Personalmente ritengo che delle tre componenti, quello della ricerca sia quello trainante e che genera la qualità e l'innovazione negli altri campi. A vedere le proposte di articoli che arrivano alla Rivista, non mi pare che i talebani della ricerca fine a se stessa siano una porzione significativa della nostra accademia di marketing. Anzi, vedo molti giovani che fanno della ricerca il proprio vettore di crescita culturale e professionale e che si dedicano responsabilmente anche alla divulgazione e all'implementazione operativa di ciò che studiano. Condivido con te che il rischio di 
autoreferenzialità sia alto, anche per atteggiamenti e comportamenti talvolta strumentali e finalizzati in chiave di carriera, ma il grosso dei colleghi mi sembra saggiamente orientato verso un sano equilibrio tra i tre pilastri di cui sopra.

Mattiacci. Pienamente d'accordo anche su questo, tuttavia mi pare ci siano ragioni per accendere un warning sulla didattica. Il nuovo sistema concorsuale su due livelli a me personalmente piace, è da affinare, ma funziona, salvo un punto: ci siamo accorti che è praticamente sparita la valutazione delle capacità didattiche del candidato? Siamo consapevoli che viviamo nel teorema del "se sai, sai anche insegnare?".

Dalli. Quali sono le sfide sul versante della didattica che $i$ docenti di marketing dovrebbero affrontare e come possiamo aiutarli?

Mattiacci. Come ho scritto nel numero speciale della Rivista per il decennale del Convegno, vedo un quadro denso di minacce per la professione del docente di marketing. Non ripeterò cose che lì ho scritto, salvo ribadire che, in sintesi estrema, secondo me noi si deve lavorare per riposizionarci in uno spazio non contendibile da chi pratica la docenza di marketing, ma non è un professore universitario.

Le nostre aule, poi, sono popolate da persone diverse da quelle che vi erano cinque anni fa, molto diverse, quasi degli alieni a confronto: ce ne siamo accorti? Ne abbiamo studiato i processi cognitivi? E quelli percettivi? Ne conosciamo le molle motivazionali all'apprendimento? Io credo che la SIMktg dovrebbe lanciare un importante progetto di ricerca sulla nuova pedagogia del marketing (e del management in generale): la Rivista potrebbe lanciarsi, per accendere l'attenzione sul tema, in un numero monografico sull'argomento, invitando colleghi pedagoghi e tecnologi a confrontarsi con noi. Come scrisse Howard Gardner, acutissimo studioso di cose umane, il paradosso di ogni Istituzione formativa è che essa procede con una struttura didattica che è espressione di scelte del passato e che questa struttura forma persone (che non ci somigliano più) che avranno modo di utilizzarne i frutti nel futuro. Dobbiamo essere consapevoli che, ogni giorno, il nostro tempo mentale di riferimento è il futuro: secondo me, sul piano culturale, questa è la più grande sfida che come formatori abbiamo di fronte.

Dalli. In effetti questa esigenza la sentono in molti anche perché il tradizionale rapporto tra docente universitario e studente non è esattamente quello che insegniamo nei nostri corsi, ovvero una relazione di fornitoreutente di un servizio. Il fornitore dovrebbe basare la propria offerta sulle esigenze dell'utente, no? Il problema che vedo, almeno nella mia sede e in quelle in cui mi capita di insegnare, è che gli stessi studenti sono scarsamente consapevoli di ciò che potrebbe o dovrebbe servire loro. Anche in questo campo mi sembra che la domanda vada al traino dell'offerta. Forse è maturo il tempo per un confronto inter-disciplinare su come e cosa si in- 
segna nelle scuole di management: ad esempio, in alcuni paesi scandinavi si stanno letteralmente smontando i dipartimenti di management generalisti per creare strutture interdisciplinari con ingegneri, umanisti, scienziati sociali e studiosi di design. Non dico che questa sia una soluzione praticabile, ma ripensare l'organizzazione culturale dei nostri programmi potrebbe essere utile.

Dalli. E la ricerca? Dal tuo punto di osservazione, quali sono le tendenze e le criticità? Cosa possono fare la Simktg e la Rivista per sostenere il lavoro dei colleghi?

Mattiacci. L'esperienza di servizio concorsuale che sto vivendo mostra che la ricerca italiana sul marketing gode di ottima salute, ma un po' precaria.

L'ottima salute si evince dalla quantità di studi e ricerche realizzate sui temi del mercato e della competitività, moltissimi dei quali trovano collocazione in eccellenti sedi internazionali (o sarebbe meglio dire, per gioco e provocazione intellettuale, angloamericane?). Credo di poter affermare che per la nostra piccola comunità l'internazionalizzazione non sia più un tema aperto, ma un fatto totalmente acquisito. Naturalmente, che poi si finisca per pubblicare su Journal of Marketing o su una rivista di minore ranking, dipende anche da fattori di contesto nei quali il ricercatore si trova a operare. Tuttavia il quadro, qui, è denso di luci più che di ombre.

Internazionalizzare la ricerca, purtroppo, conferisce un valore drammatico al tema delle risorse disponibili: costruire e mantenere quelle necessarie relazioni, che poi servono a darsi un respiro non solo domestico, costa e costa tanto. Questa è una prima ragione della precarietà che dicevo prima. La seconda è la rilevanza: onestamente, se fossi un manager o un imprenditore, sarei interessato a meno del dieci percento dei lavori che ho visto sottoporre a valutazione. Personalmente credo che il portare la ricerca su temi estetici ed esercizi di stile, come purtroppo ogni tanto avviene, ci condurrà in una zona buia, passando prima, ogni tanto, per il ridicolo.

Dalli. Allora, se mi permetti una piccola provocazione, ci sarebbe da lavorare anche sui manager e sugli imprenditori di questo paese. È vero che talvolta alcuni colleghi tendono all'esercizio di stile, ma è anche vero che il livello di reale interesse e di preparazione di alcuni professionisti lascia alquanto a desiderare e che spesso si tende ad appiattire il rapporto con la ricerca universitaria su di un piano meramente commerciale. In alcuni paesi stranieri, ad esempio, sono assai frequenti i casi di professionisti con titoli di Mba e di Phd, cosa che da noi è quanto mai infrequente. Alla base di una certa distanza tra università e business c'è anche questo gap di natura tecnica e culturale.

Mattiacci. Sembra che ci si sia messi d'accordo: sono totalmente dello stesso parere. In questo Paese abbiamo una classe imprenditoriale, mana- 
geriale e dirigente che lascia molto a desiderare sul piano della cultura personale, dell'aggiornamento e della qualificazione. Hai voglia a denunciare sui giornali i mali dell'Università, ma se i giovani laureati non trovano lavoro, è anche perché non abbiamo gente capace o desiderosa di creare posti di lavoro qualificati.

Dalli. Infine, come facciamo a riqualificare il nostro ruolo come docenti e ricercatori universitari di marketing? La Simktg e la Rivista lavorano anche per questo, ma ai colleghi non vogliamo dare qualche indicazione e qualche incoraggiamento?

Mattiacci. Anche in questo caso rischio di ripetermi rispetto a quanto scritto nel numero speciale del decennale del convegno. Per farla breve, e semplice, io credo che il professore universitario sia e debba essere un intellettuale, non (non solo perlomeno) un tecnico. I tecnici stanno a scuola, le Università devono essere popolate di intellettuali. Un intellettuale possiede e nutre la propria cultura e questa entità, in costante trasformazione, gli conferisce un modo di vedere le cose personale, unico e originale. Un tecnico racconta le cose che altri hanno pensato (magari si appoggia anche a slide fatte da altri) e, secondo me, per lui in Università non c'è posto. Un intellettuale accetta e ricerca il confronto di idee e si preoccupa di esprimere il suo pensiero in tutti i modi e le forme possibili, perché ha e deve avere, l'ambizione di cambiare il mondo.

Essere intellettuale significa collocarsi nella posizione di accettare il divenire costante del proprio sapere, significa farsi partecipe della realtà del proprio tempo nei modi che sono consoni al proprio tempo, cercando di modificarla in senso migliorativo.

Io credo che noi ci si debba sforzare di recuperare alla professione il proprio lato intellettuale: ci vuole tempo e fatica, ma dobbiamo farlo.

Dalli. Un ricercatore, docente e" riformatore", insomma. Una tradizione di pensiero che nella nostra comunità è assai radicata, sia nell'ambito del marketing che nel settore di Economia e gestione delle imprese più in generale.

Mattiacci. Infine un aspetto più leggero, ma non meno rilevante. Quando leggo su un grande quotidiano nazionale che l'opinione sulle strategie di mercato della Fiat le chiedono a un economista, o a un sociologo, penso che finora abbiamo fallito la nostra missione di intellettuali, lasciando questo ruolo ad altri, non necessariamente più qualificati in materia di noi. Sarà cura della SIMktg nell'immediato futuro contribuire a superare questo stato di cose.

Dalli. Su questo purtroppo non possiamo che fare autocritica e forse noi di marketing più di altri. Se è vero infatti che questa tua ultima lamentela è condivisa anche da altri aziendalisti che in Aidea vedo ben rappre- 
sentati, chi si occupa di marketing ha il dovere di investire in modo più efficace sulla propria immagine e sul proprio posizionamento, intellettuale e professionale. Per convincere i decisori nelle imprese e nelle istituzioni ad ascoltare il proprio punto di vista. Sembra un paradosso, ma se proprio noi non riusciamo a comunicare il valore aggiunto che siamo in grado di mettere a disposizione della società, allora non siamo tanto bravi come docenti di marketing. Da questo punto di vista la SIMktg e la Rivista stanno lavorando per far crescere la visibilità e il credito di cui gode la comunità dei docenti di marketing, ma anche questi ultimi devono darsi da fare per sfruttare al meglio le opportunità che sono a loro disposizione.

Dalli. Caro Alberto, a nome della Rivista ti ringrazio del tempo che ci hai concesso e dell'onestà intellettuale con cui sostieni i tuoi punti di vista. Sono convinto che i lettori apprezzeranno come me il tuo sforzo di metterti a disposizione dell'Accademia nell'interesse dell'accademia.

Pisa, Roma

Ottobre 2013 